\title{
Revisiting Lévy flight search patterns of wandering albatrosses, bumblebees and deer
}

Andrew M. Edwards ${ }^{1, *}$, Richard A. Phillips ${ }^{1}$, Nicholas W. Watkins ${ }^{1}$, Mervyn P. Freeman ${ }^{1}$, Eugene J. Murphy ${ }^{1}$, Vsevolod Afanasyev ${ }^{1}$, Sergey V. Buldyrev ${ }^{2,3}$, Marcos G. E. da Luz ${ }^{4}$, Ernesto P. Raposo ${ }^{5}$, H. Eugene Stanley ${ }^{2}$ \& Gandhimohan M. Viswanathan 6

${ }^{1}$ British Antarctic Survey, High Cross, Madingley Road, Cambridge, CB3 OET, $U K$

${ }^{2}$ Center for Polymer Studies and Department of Physics, Boston University, Boston, MA 02215, USA

${ }^{3}$ Department of Physics, Yeshiva University, 500 West 185th Street, Room 1112, Belfer Hall, New York, NY 10033, USA

${ }^{4}$ Departamento de Física, Universidade Federal do Paraná, C. P. 19044, 81531990, Curitiba-PR, Brazil

${ }^{5}$ Laboratório de Física Teórica e Computacional, Departamento de Física, Universidade Federal de Pernambuco, 50670-901, Recife-PE, Brazil

${ }^{6}$ Instituto de Física, Universidade Federal de Alagoas, 57072-970, Maceió-AL, Brazil 
${ }^{*}$ Current address: Pacific Biological Station, Fisheries and Oceans Canada, 3190 Hammond Bay Road, Nanaimo, BC, V9T 6N7, Canada

The study of animal foraging behaviour is of practical ecological importance ${ }^{1}$, and exemplifies the wider scientific problem of optimizing search strategies ${ }^{2}$. Lévy flights are random walks whose step lengths come from probability distributions with heavy power-law tails ${ }^{3,4}$, such that clusters of short steps are connected by rare long steps. They display fractal properties, have no typical scale, and occur in physical ${ }^{3-5}$ and chemical ${ }^{6}$ systems. An attempt to demonstrate their existence in a natural biological system presented evidence that wandering albatrosses perform Lévy flights when searching for prey on the ocean surface ${ }^{7}$. This well-known finding ${ }^{2,4,8,9}$ was followed by similar inferences about search strategies of deer $^{10}$ and bumblebees ${ }^{10}$. These pioneering studies have triggered much theoretical work in physics (e.g. refs 11, 12), and empirical ecological analyses regarding reindeer $^{13}$, microzooplankton ${ }^{14}$, grey seals ${ }^{15}$, spider monkeys ${ }^{16}$ and fishing boats ${ }^{17}$. Here we analyze a new, high-resolution data set of wandering albatross flights, and find no evidence for Lévy flight behaviour. Instead we find that flight times are gamma distributed, with an exponential decay for the longest flights. We re-analyze the original albatross data ${ }^{7}$ using additional information, and conclude that 
the extremely long flights, essential for demonstrating Lévy flight behaviour, were spurious. Furthermore, we propose a widely applicable method to test for power-law distributions using likelihood ${ }^{18}$ and Akaike weights ${ }^{19,20}$. We apply this to the four original deer and bumblebee data $\operatorname{sets}^{10}$, finding that none exhibit evidence of Lévy flights, and that the original graphical approach ${ }^{10}$ is insufficient. Such a graphical approach has been adopted to conclude Lévy flight movement for other organisms ${ }^{13-17}$, and to propose Lévy flight analysis as a potential real-time ecosystem monitoring tool ${ }^{17}$. Our results question the strength of the empirical evidence for biological Lévy flights.

In 1992, five wandering albatrosses (Diomedea exulans) on Bird Island, South Georgia $\left(54^{\circ} 00^{\prime} \mathrm{S}, 38^{\circ} 03^{\prime} \mathrm{W}\right)$ each had a salt-water immersion logger ${ }^{21}$ attached to one of its legs. Over the course of a bird's foraging trip, the logger recorded the proportion of each hour spent sitting on the sea surface. In ref. 7, flight durations (time intervals between landing on the ocean) were then calculated as consecutive hours for which a bird remained dry, to a resolution of $1 \mathrm{~h}$. It was assumed that birds landed on the water solely to feed, and that flight durations were thus indicative of distances between prey.

Time series for 19 separate foraging trips ${ }^{7}$ were pooled to give a total of 363 
flights. The resulting log-log histogram of flight durations gave a straight line with a slope of approximately 2, and is reproduced in Supplementary Fig. 1 from the original raw data. The crux of the conclusion that the albatrosses were performing Lévy flights was that the slope of 2 implied the probability density function (pdf) of flight durations $t$ (in hours), was ${ }^{7,10}$

$$
f(t) \sim t^{-2}
$$

for $t \geq 1 \mathrm{~h}$ (leaving out the normalization constant). This is consistent with the Lévy flight definition that the tail of the pdf is of the power-law form $t^{-\mu}$, where $1<\mu \leq 3$ (though technically this is a Lévy walk ${ }^{4,7,22}$ ). The Lévy flight was inferred to be an efficient foraging strategy for food that might be fractally distributed on the ocean surface ${ }^{7}$.

We first analyze a newer, larger, and higher resolution data set of albatross flight durations to test for Lévy flights. In 2004, 20 wandering albatrosses on Bird Island were each fitted with a salt-water logger and a GPS device. The GPS data were too infrequent (at most one location $\mathrm{h}^{-1}$ ) to give distances between landings, but were needed to estimate each bird's departure time from Bird Island, in order to calculate the duration of the initial flight before first landing on the water (we calculated return flights similarly). The resulting data set of flight records was 
pooled, as in ref. 7, yielding a total of 1416 flights to a resolution of $10 \mathrm{~s}$ (Fig. 1).

The flights $\geq 1 \mathrm{~h}$ are clearly inconsistent with coming from the power law $t^{-2}$ ascertained $^{7}$ for the 1992 data. Furthermore, data from a power law of any exponent (not just 2) would yield a straight line ${ }^{23}$, and this is clearly not the case. In fact, the flight durations $t$ (in $\mathrm{h}$ ) are consistent with coming from the shifted gamma distribution given by the pdf

$$
f(t)=\frac{r^{s}}{\Gamma(s)} y^{s-1} \mathrm{e}^{-r y}
$$

where $y=t-1 / 120$ accounts for the assumed $30 \mathrm{~s}$ period before the bird searches for new food sources (see Methods), $s=0.31$ is the shape parameter, $r=0.41 \mathrm{~h}^{-1}$ is the rate parameter, and $\Gamma(\cdot)$ is the gamma function. Equation (2) is valid for flights $>30 \mathrm{~s}$; for shorter flights we have $f(t)=0$. The exponential term of (2) dominates for large $t$, implying Poisson behaviour, such that for long enough flights the birds essentially encounter prey randomly with a constant low probability.

A Brownian random walker's displacement increases as $t^{H}$ where $H=1 / 2$. If $H>1 / 2$, we have "superdiffusion" as originally inferred in Fig. 2a of ref. 7. Superdiffusion is explained by one or both of the following ${ }^{3,4}$ : (i) the pdf of flight 
lengths has a heavy tail, or (ii) there is long range persistence in direction or time. The term Lévy flight is usually reserved ${ }^{4}$ for case (i). Ref. 7 used a Lévy walk model that assumes constant velocity, yielding a power law pdf of flight durations. The gamma distribution (2) has $\mu=1-s=0.69$. This is such a slow powerlaw decay that it is non-normalizable unless there is truncation. Hence, unlike the truncated Lévy flight ${ }^{3}$ where $1<\mu \leq 3$, equation (2) cannot be interpreted as a power law with exponential truncation. Superdiffusion remains possible, but through directional persistence only, not Lévy flights.

The longest flight in 2004 was 14.9 h, whereas for the original 1992 data $^{7}$, 25 of the 363 flights were $>15$ h. In 1992, for six of the trips the birds were also fitted with a satellite transmitter (Platform Terminal Transmitter, PTT) which provided locations at irregular intervals. While these data are also too coarse to determine flight distances between landings (and were not available for ref. 7), we use them here to determine when each bird left and returned to Bird Island (see Methods). Figure 2 shows the wet/dry data for these six trips, together with the estimated departure and return times based on the PTT data. For trip 3B, for example, the first dry sequence, based solely on the salt-water-logger data, is $46 \mathrm{~h}$. However, the PTT data reveal that the bird did not leave Bird Island until $41 \mathrm{~h}$ after 
the logger was switched on. Thus the true duration of the first flight was only $5 \mathrm{~h}$.

For the remaining five trips, the original dry sequences from the loggers (in the order of Fig. 2) of 44, 69, 26, 67 and 23 h, represent, in reality, flight records of only $4,3,1,<1$ and $<1 \mathrm{~h}$, respectively. Similarly, for final flights the raw logger data values of $4,8,13,9,34$ and $9 \mathrm{~h}$, get corrected to true flight records of $4,5,8,2,3$ and $7 \mathrm{~h}$, respectively.

However, in ref. 7 the raw logger data were assumed to represent true flights. We adjust the data for the remaining 13 trips, for which no PTT data were collected, by eliminating the initial and final dry sequences (see Methods). Using these adjusted data, in Fig. 3a we compute a corrected version of the original loglog histogram shown in Fig. 3a of ref. 7. There are now no flight durations in the two largest bins, and the longest flight is only $20 \mathrm{~h}$ compared to the original $99 \mathrm{~h}$. The data thus no longer span two orders of magnitude, and the points clearly lie on a curve, not a straight line that would indicate a power law.

The data are also consistent with coming from a shifted gamma distribution of the form (2), illustrated in Fig. 3b. The resulting confidence intervals for $s$ and $r$ are much larger than those for the 2004 data, because of the smaller sample 
size, lack of data $<1 \mathrm{~h}$, and lower resolution of the data. Figure $3 \mathbf{c}$ shows the uncorrected and corrected data as a rank/frequency plot, as in Fig. 1. We conclude that, when time spent by the birds on the nests is accounted for, the original 1992 albatross data do not support Lévy flight behaviour.

The original albatross study ${ }^{7}$ was followed by reports of Lévy flight behaviour by deer (Dama dama $)^{10}$ and bumblebees (Bombus terricola $)^{10}$. The deer data were plotted as a standard histogram log-transformed (LT in terminology of ref. 24), the bees data as a smoothed histogram log-transformed ${ }^{10}$, and straight lines were compared to the tails. The problematic ${ }^{24,25}$ LT method was then used to conclude Lévy flight behaviour in other studies ${ }^{13,15-17,26}$, and the LBN method used in ref. 14. In no cases were alternative distributions properly considered, or goodness-of-fit tests performed. In Box 1 we present a new method to overcome these shortcomings. We now illustrate this approach by re-analyzing the deer and bumblebee data ${ }^{10}$.

Following ref. 10, we digitized data from ref. 27 concerning foraging times of deer in unfenced and fenced areas, and digitized data from ref. 28 concerning flights of bumblebees between flower-heads in high- and low-food situations. In ref. 10 these data were all assumed to relate to distances travelled between food 
items. Likelihood functions modified from those in Box 1 are calculated in the Supplementary Information (requiring numerical maximization), to account for the data only being available already binned. The resulting Akaike weights $w_{1}$ for the unbounded power-law tail were $<10^{-8}$ for both deer data sets, and 0.40 and 0.001 for the bees (and for the 0.40 case, $\mu$ is outside the Lévy range). Given such negligible support for the unbounded power law in the Lévy range, we also tested both models over the bounded ranges $[a, b]$ assumed in ref. 10; see Table 1 .

We find that for the deer scenarios the exponential distribution is favoured by Akaike weights of 0.9994 and 0.95 . Furthermore, the reported power-law $\mu$ values $^{10}$ lie outside the $95 \%$ CIs for the MLE values, and are inconsistent with the data. Figure 4 shows the log-log histograms of the deer data and reported power laws from ref. 10, plus the exponential distributions calculated in Table 1. By eye the two distributions do not appear as different as concluded statistically. However, the log-log nature of Fig. 4 acts to minimize (to the eye) any differences, and de-emphasizes the fact that there are far more data represented by some points (bins) than others. This illustrates the problems involved with fitting lines (or curves) to histograms plotted on a log-log scale.

For the high-food bees the Akaike weights essentially favour neither model 
(Table 1). For the low-food bees the power law is favoured, but not convincingly. However, we have assumed maximum attainable flight values (b) based simply on the ranges of the power-law lines drawn in ref. 10. This assumption favours preference for the power law, and relaxing it slightly (since it is very unlikely to hold) eliminates the marginal preference for the power law in the low-food bees scenario (see Supplementary Information). Hence, none of the four data sets exhibit evidence for the power law over the exponential.

Furthermore, the deer foraging times ${ }^{10}$ actually correspond to times spent cropping and processing food (i.e. handling times) at a particular feeding site (pages 608 and 610 in ref. 27), rather than time spent moving between sites. Also the bumblebee data actually represent the distances between successively visited flower heads, rather than the flight times or distances flown (Fig. 1 in ref. 28). Therefore, neither of these data sets was ideal for testing for Lévy flight search behaviour. Nonetheless, we have re-analyzed these data sets to demonstrate the problems with inferring Lévy flight behaviour by fitting (or drawing) a straight line through points on a log-log histogram, not considering alternative distributions, and not performing goodness-of-fit tests. Our approach used here is grounded in the statistical theory of likelihood ${ }^{18,19}$, avoids any binning, and does 
not ignore bins with zero counts ${ }^{25}$. Given these issues relating to the identification of power laws, we query whether the current evidence for Lévy flights would withstand more rigorous statistical analysis. This then raises questions as to when might a foraging animal satisfy the necessary conditions for a Lévy flight to be an optimal $^{2,10}$ search strategy.

\section{METHODS SUMMARY}

Maximum likelihood estimates were obtained by numerically maximizing the appropriate log-likelihood functions, and 95\% CIs were computed using the profile likelihood-ratio test ${ }^{18}$. All goodness-of-fit tests were performed using the G-test (likelihood-ratio test) with Williams's correction ${ }^{29}$. Akaike weights were computed according to ref. 19, with bias-adjusted $\mathrm{AIC}_{\mathrm{c}}$ used for high-food bees due to the small sample size. Computations were performed using $\mathrm{R}$, version 2.2.0 (www.r-project.org).

Full Methods are available in the online version of the paper at www.nature.com/nature.

\section{METHODS}

Initial and final albatross flights. A salt-water logger only detects whether a 
bird is sitting on the water or not, and its clock starts when it is switched on at a computer. Thus a logger is recording before being attached to a bird, and also, crucially, while the bird sits on a nest ${ }^{21}$. The logger is dry, but the bird is not flying. So the initial sequence of dry readings includes pre-take-off time plus time spent in flight before first landing on water. For the 2004 data we used the GPS data to determine when the birds left Bird Island, and hence obtained the duration of the initial flights (to within an hour), eliminating the time spent on the nest. Return times to the island were used to determine durations of final flights, eliminating the dry period between landing and logger retrieval.

In 1992, PTT devices were used in 6 of the 19 trips (Fig. 2). Departure and return times were calculated to within $2 \mathrm{~h}$ (typically $1 \mathrm{~h}$ ) from a combination of the PTT fixes and direct observations of the birds. For the remaining 13 trips, departure and return times remain unknown. As each trip's initial and final dry sequences were potentially spurious, we omitted them from the data. If the albatrosses really exhibited Lévy flight behaviour then there would be nothing special about the first and last flights, so eliminating these 26 dry sequences should not markedly impact the results. However, the 14 longest dry sequences (for the 13 trips), ranging from 21-99 $\mathrm{h}$, all occurred at the start or end of trips and were 
eliminated. These were much longer than the maximum flight of $14.9 \mathrm{~h}$ in 2004.

Albatross data analysis. The 2004 data consisted of a wet/dry reading every $10 \mathrm{~s}$. A flight is therefore represented by a sequence of consecutive dry readings in between two wet readings. For example, a sequence wet-dry-dry-dry-dry-wet gives a record of 4 dry readings, and represents a flight in the range $30-50 \mathrm{~s}$. We assume $30 \mathrm{~s}$ to be the minimum time after take off for a bird to start searching for new food sources. This excludes instances where a bird may have only lifted its leg out of the water to scratch (yielding a sequence wet-dry-wet), or abandoned a take off.

The 1992 data consisted of the number of $15 \mathrm{~s}$ intervals within each hour for which a bird was considered wet (e.g. Fig. 1 of ref. 7). Flights $<1$ h could not be directly inferred from the data. In ref. 7, consecutive hourly wet counts of 0 (i.e. fully dry hours), in between non-zero hours, were used to infer flight times of $1,2,3, \ldots \mathrm{h}$. A sequence of hours wet-dry-wet was then assumed to be a flight of $1 \mathrm{~h}$. However, such a record could come from a true flight anywhere in the range 1-3 h. In the Supplementary Information we derive the likelihood functions for each data set, taking into account the respective sampling protocols. 
Deer and bumblebee data analysis. We digitized the deer and bumblebee data from the original histograms ${ }^{27,28}$ and from ref. 10. For the deer data the original linear histograms ${ }^{27}$ were log-transformed ${ }^{10}\left(\mathrm{LT}\right.$ method $\left.^{24}\right)$, as reproduced in Fig. 4 from the original data ${ }^{27}$. Whereas the bumblebee bins $^{28}$ were smoothed using running averaging and then lumped to produce log-log histograms ${ }^{10}$ (see Supplementary Information). However, our likelihood approach and results in Table 1 do not involve any smoothing or lumping of the original binned data.

We fit the power-law and exponential distributions over unbounded, $[a, \infty)$, and bounded, $[a, b]$, ranges. For the bounded power law we consider the pdf

$$
f(x)=C x^{-\mu}, \quad x \in[a, b]
$$

where $C$ is the normalization constant given by $C=(\mu-1) /\left(a^{1-\mu}-b^{1-\mu}\right)$. For the bounded exponential distribution, we have

$$
f(x)=A \mathrm{e}^{-\lambda x}, \quad x \in[a, b]
$$

where $A$ is the normalization constant given by $A=\lambda /\left(\mathrm{e}^{-\lambda a}-\mathrm{e}^{-\lambda b}\right)$. The data were digitized from histograms ${ }^{27,28}$, and so are already binned. In the Supplementary Information we derive the likelihood functions for each distribution, modified from those in Box 1 to account for the pre-binned data and the bounded ranges. 
For each scenario in Table 1 , we took $[a, b]$ to be the ranges implied by the powerlaw straight lines drawn in ref. 10. These ranges imply that it is impossible to obtain data larger than that observed, an assumption that is very unlikely to be true in practice, but that we find favours preference for the power law over the exponential. We investigate sensitivity to $b$ in the Supplementary Information, showing that the result of the power law being slightly favoured for the low-food bees (Table 1) is not robust to relaxation of this assumption.

1. Turchin, P. Quantitative Analysis of Movement: Measuring and Modeling Population Redistribution in Animals and Plants (Sinauer Associates, Sunderland, MA, 1998).

2. Shlesinger, M. F. Search research. Nature 443, 281-282 (2006).

3. Shlesinger, M. F., Zaslavsky, G. M. \& Frisch, U. (eds.) Lévy Flights and Related Topics in Physics (Springer-Verlag, Berlin Heidelberg, 1995).

4. ben-Avraham, D. \& Havlin, S. Diffusion and Reactions in Fractals and Disordered Systems (Cambridge University Press, Cambridge, 2000).

5. Bardou, F., Bouchaud, J.-P., Aspect, A. \& Cohen-Tannoudji, C. Lévy Statistics and Laser Cooling: How Rare Events Bring Atoms to Rest (Cambridge 
University Press, Cambridge, 2002).

6. Ott, A., Bouchaud, J. P., Langevin, D. \& Urbach, W. Anomalous diffusion in "living polymers": a genuine Lévy flight? Phys. Rev. Lett. 65, 2201-2204 (1990).

7. Viswanathan, G. M. et al. Lévy flight search patterns of wandering albatrosses. Nature 381, 413-415 (1996).

8. Metzler, R. \& Klafter, J. The restaurant at the end of the random walk: recent developments in the description of anomalous transport by fractional dynamics. J. Phys. A: Math. Gen. 37, R161-R208 (2004).

9. Klafter, J. \& Sokolov, I. M. Anomalous diffusion spreads its wings. Phys. World 18, 29-32 (2005).

10. Viswanathan, G. M. et al. Optimizing the success of random searches. Nature 401, 911-914 (1999).

11. Reynolds, A. M. Scale-free movement patterns arising from olfactory-driven foraging. Phys. Rev. E 72, 041928 (2005). 
12. Bénichou, O., Loverdo, C., Moreau, M. \& Voituriez, R. Two-dimensional intermittent search processes: An alternative to Lévy flight strategies. Phys. Rev. E 74, 020102(R) (2006).

13. Mårell, A., Ball, J. P. \& Hofgaard, A. Foraging and movement paths of female reindeer: insights from fractal analysis, correlated random walks, and Lévy flights. Can. J. Zool. 80, 854-865 (2002).

14. Bartumeus, F., Peters, F., Pueyo, S., Marrasé, C. \& Catalan, J. Helical Lévy walks: Adjusting searching statistics to resource availability in microzooplankton. PNAS 100, 12771-12775 (2003).

15. Austin, D., Bowen, W. D. \& McMillan, J. I. Intraspecific variation in movement patterns: modeling individual behaviour in a large marine predator. Oikos 105, 15-30 (2004).

16. Ramos-Fernández, G. et al. Lévy walk patterns in the foraging movements of spider monkeys (Ateles geoffroyi). Behav. Ecol. Sociobiol. 55, 223-230 (2004).

17. Bertrand, S., Burgos, J. M., Gerlotto, F. \& Atiquipa, J. Lévy trajectories of Peruvian purse-seiners as an indicator of the spatial distribution of anchovy (Engraulis ringens). ICES J. Mar. Sci. 62, 477-482 (2005). 
18. Hilborn, R. \& Mangel, M. The Ecological Detective: Confronting Models with Data (Vol. 28, Monographs in Population Biology, Princeton University Press, New Jersey, 1997).

19. Burnham, K. P. \& Anderson, D. R. Model Selection and Multimodel Inference: A Practical Information-Theoretic Approach (2nd ed., Springer, New York, 2002).

20. Johnson, J. B. \& Omland, K. S. Model selection in ecology and evolution. Trends Ecol. Evol. 19, 101-108 (2004).

21. Afanasyev, V. A miniature storing activity recorder for seabird species with 80 bytes of memory for data storage. NERC Tech. 1, 4-7 (1993).

22. Shlesinger, M. F. \& Klafter, J. Lévy walks versus Lévy flights. In Stanley, H. E. \& Ostrowsky, N. (eds.) On Growth and Form: Fractals and Non-Fractal Patterns in Physics, 279-283 (Martinus Nijhoff Publishers, Dordrecht, 1986).

23. Newman, M. E. J. Power laws, Pareto distributions and Zipf's law. Contemp. Phys. 46, 323-351 (2005).

24. Sims, D. W., Righton, D. R. \& Pitchford, J. W. Minimizing errors in identifying Lévy flight behaviour of organisms. J. Anim. Ecol. 76, 222-229 (2007). 
25. Pueyo, S. \& Jovani, R. Comment on "A keystone mutualism drives pattern in a power function”. Science 313, 1739c (2006).

26. Weimerskirch, H., Gault, A. \& Cherel, Y. Prey distribution and patchiness: factors in foraging success and efficiency of wandering albatrosses. Ecology 86, 2611-2622 (2005).

27. Focardi, S., Marcellini, P. \& Montanaro, P. Do ungulates exhibit a food density threshold? A field study of optimal foraging and movement patterns. J. Anim. Ecol. 65, 606-620 (1996).

28. Heinrich, B. Resource heterogeneity and patterns of movement in foraging bumblebees. Oecologia 40, 235-245 (1979).

29. Sokal, R. R. \& Rohlf, F. J. Biometry: The Principles and Practice of Statistics in Biological Research (3rd ed., W. H. Freeman and Company, New York, 1995).

30. Pueyo, S. Diversity: between neutrality and structure. Oikos 112, 392-405 (2006).

Supplementary Information accompanies the paper on www.nature.com/nature. 
Acknowledgements We thank Peter Rothery, Wade Blanchard and Len Thomas for statistical advice, and Ransom Myers, Ian Jonsen, Greg Breed, Sara-Jane Dunn, Francisco de Moura, José Cressoni and Marcelo Lyra for discussions. We acknowledge the work by all fieldworkers involved, in particular thanking Ben Phalan and Isaac Forster for deploying devices at Bird Island. We thank Mike Francis, Akira Fukuda and Hiroyoshi Higuchi for providing instruments used in 2004, and John Croxall for supporting albatross research at Bird Island. This work was funded by the UK Natural Environment Research Council and the Brazilian research agency CNPq. The work at the British Antarctic Survey represents a collaboration between the Discovery 2010 and Natural Complexity Programmes, and we appreciate Phil Trathan's efforts in facilitating it.

Authors' Contributions AME performed the analyses, computations and derivations presented in this paper, and led its preparation with input from all authors. The paper presents a synthesis of the work of two different teams of researchers who independently and concurrently conceived of re-examining the Lévy flight hypothesis with newer albatross data. One team (A) comprised NWW, MPF and EJM and was subsequently joined by AME. The other team (B) comprised VA, SVB, MGEdL, EPR, HES and GMV. RAP provided albatross expertise to both teams. Team B were the first to show the implication of the long first and last dry sequences on the power law distribution of albatross flight durations. 
Competing Interests The authors declare that they have no competing financial interests.

Correspondence Correspondence and requests for materials should be addressed to AME (email: EdwardsAnd@pac.dfo-mpo.gc.ca). 
Re-analysis of data sets for which Lévy flight behaviour was concluded ${ }^{10}$

\begin{tabular}{|c|c|c|c|c|c|}
\hline \multirow[t]{3}{*}{ Data set } & \multirow{3}{*}{$\begin{array}{c}\text { Reported } \\
\mu\end{array}$} & \multirow{3}{*}{$\begin{array}{c}\text { MLE for } \mu \\
(95 \% \mathrm{Cl}) \\
\quad G, p\end{array}$} & \multirow{3}{*}{$\begin{array}{c}\text { MLE for } \lambda \\
(95 \% \mathrm{Cl}) \\
\quad G, p\end{array}$} & \multicolumn{2}{|c|}{ Akaike weights } \\
\hline & & & & Power law & Exponential \\
\hline & & & & & \\
\hline \multirow{3}{*}{$\begin{array}{l}\text { Deer, } \\
\text { unfenced }\end{array}$} & 2.0 & 1.57 & 0.0264 & 0.0006 & 0.9994 \\
\hline & & $(1.29,1.85)$ & $(0.0214,0.0318)$ & & \\
\hline & & $19.0,0.0003$ & $4.2,0.24$ & & \\
\hline \multirow{3}{*}{$\begin{array}{l}\text { Deer, } \\
\text { fenced }\end{array}$} & 2.1 & 1.44 & 0.0290 & 0.05 & 0.95 \\
\hline & & $(1.06,1.83)$ & $(0.0212,0.0372)$ & & \\
\hline & & $4.87,0.088$ & $1.08,0.58$ & & \\
\hline \multirow{3}{*}{$\begin{array}{l}\text { Bees, } \\
\text { high food }\end{array}$} & 3.5 & 3.68 & 0.0153 & 0.59 & 0.41 \\
\hline & & $(2.14,5.42)$ & $(0.0086,0.0234)$ & & \\
\hline & & $0.16,0.69$ & $0.59,0.44$ & & \\
\hline \multirow{3}{*}{$\begin{array}{l}\text { Bees, } \\
\text { low food }\end{array}$} & 2.0 & 2.20 & 0.00609 & 0.78 & 0.22 \\
\hline & & $(1.84,2.60)$ & $(0.00497,0.00732)$ & & \\
\hline & & $11.3,0.19$ & $13.1,0.11$ & & \\
\hline
\end{tabular}

Table 1: 


\section{Table and Figure Captions}

\section{Table 1. Re-analysis of data sets for which Lévy flight behaviour was concluded ${ }^{10}$.}

Tests are over bounded ranges $[a, b]$, with $a, b$ and reported power-law exponents for $\mu$ taken from ref. 10. Goodness-of-fit values $G$ and $p$ are for the G-test ${ }^{29}$ ( $n$ and df for the four scenarios are, respectively, 141 and 3, 112 and 2, 25 and 1, and 129 and 8). For both deer scenarios, the reported $\mu$ values ${ }^{10}$ lie outside the $95 \%$ CIs. For the unfenced deer, the data are inconsistent with coming from a power law at the MLE value (and so are certainly inconsistent with the reported $\mu$, for which we calculate $G=27.9, p<10^{-5}$ ). The Akaike weights overwhelmingly favour the exponential. For the fenced deer, the data are consistent with coming from a power law with the MLE exponent of $\mu=1.44$ (though not with the reported exponent of $\mu=2.1$, for which $G=16.7, p=0.0002)$. The Akaike weights favour the exponential fairly convincingly. For the high-food bees the data were considered to follow a power law ${ }^{10}$, but with the reported $\mu$ lying outside the Lévy range $1<\mu \leq 3$. The CI computed here does overlap the Lévy range, but the Akaike weights favour neither the power law or exponential. For the low-food bees the reported $\mu$ lies within the $95 \% \mathrm{CI}$, and the power law is indeed favoured (using the MLE value for $\mu$ ), but not convincingly. 
Figure 1. Rank/frequency plot $^{23}$ of 2004 wandering albatross data, showing no evidence for Lévy flight behaviour. Circles show number of flights $\geq t$ for each flight duration $t$ (calculated by ranking flights by size). Red curve is the fit to the shifted gamma distribution (2) with maximum likelihood estimates (MLEs) of $s=0.31$ (95\% Confidence Interval (CI): 0.27-0.34) and $r=0.41 \mathrm{~h}^{-1}(95 \%$ CI:0.36-0.46), obtained by maximizing the multinomial likelihood function that takes into account the discrete sampling nature of the loggers (see Supplementary Information). The data are consistent with coming from this distribution ( $n=$ 1416, $\mathrm{df}=37, G=28.9, p=0.83$ ). Flights are correct to within $\pm 10 \mathrm{~s}$ (see Supplementary Information). If the flights $\geq 1 \mathrm{~h}$ followed the power law with exponent $\mu=2$ as in ref. 7 , the points would lie on the straight blue line ${ }^{23}$ (that has been vertically shifted slightly for clarity) - this is clearly not the case. Inset shows the 2004 data as a conventional histogram on linear axes, with number of flights against flight duration in hours.

Figure 2. Data for the six wandering albatross trips in 1992 that have known departure and return times. Red lines are hours for which a logger was completely dry, blue lines indicate hours when a logger was wet for some part of that hour, and grey lines indicate switches between these regimes. Black lines indicate 
when each bird departed from and returned to Bird Island, based on the PTT data. Time 0 is when the loggers were switched on at a computer - thus intervals before the first black lines include time taken to affix the logger to a bird plus time spent by the bird sitting on its nest before departing. All birds remained on Bird Island for long periods before departing, but such periods were considered to be flights in the original study ${ }^{7}$. Intervals after the final black lines correspond to time the bird sat on its nest after returning plus time spent retrieving the logger, but these were also originally considered as flights ${ }^{7}$.

\section{Figure 3. When corrected, the 1992 wandering albatross flight durations no}

longer follow a power law. a Blue open circles show original log-log histogram of 1992 data (Fig. 3a of ref. 7). Breakpoints of bins are at 1, 2, 4, 8, 16, 32, 64 and $128 \mathrm{~h}$ (with bin intervals $1 \leq t<2,2 \leq t<4$, etc.), and results are plotted at the geometric means. The frequencies are each normalized by their respective bin widths to yield frequency densities that compensate for the increasing bin widths $^{30}$ (termed logarithmic binning with normalization, LBN, in ref. 24). The straight line indicates a power law of exponent $\mu=2$ (ref. 7). Red filled circles are adjusted flight durations that take into account time spent on Bird Island, binned

in the same manner, showing no power-law behaviour. b The gamma distribution 
fitted to the (unbinned) flight durations (red curve) has MLE values $s=0.73$ (95\% CI: $0.19-1.32)$ and $r=0.33 \mathrm{~h}^{-1}$ (95\% CI: 0.22-0.46), and the data are consistent with coming from this distribution $(n=335, \mathrm{df}=8, G=11.9, p=0.16)$. This distribution yields expected counts in each bin (black open circles), which are what should be compared with the binned data. Our multinomial likelihood approach accounts for the fact that the loggers' memory limitations meant that a record of $1 \mathrm{~h}$ could correspond to a flight anywhere in the range 1-3 $\mathrm{h}$. This fact plus the effects of the binning procedure result in the differences between the red curve and black circles (note the log scale); see Supplementary Information. c Original (blue open circles) and adjusted (red closed circles) data as a rank/frequency plot. Each record yields a point, and since the resolution of the logger data was $1 \mathrm{~h}$, there can be multiple points for each given flight duration $t$. The ordinate shows proportion rather than number of flights, because of the different sizes of the data sets.

Figure 4. Foraging times of deer previously concluded to demonstrate Lévy flight behaviour ${ }^{\mathbf{1 0}}$. a Digitized data of foraging times of unfenced deer from Fig. 3 of ref. 27, as plotted in Fig. $3 \mathrm{c}$ of ref. 10. Circles are percentages of foraging times in each bin. Bins are each $20 \mathrm{~s}$ wide, though the axes are logarithmic (the 
LT method ${ }^{24}$ ). Blue line is power law of exponent $\mu=2$ from ref. 10 , and red curve is superior fit of an exponential tail (see Table 1). b As for a, but for deer in fenced areas. Blue line is power law of exponent $\mu=2.1$ from ref. 10 , and red curve is superior fit of an exponential tail.

\section{Box 1: When is a power law not a power law?}

The approach widely used to test for biological Lévy flight search patterns has been: (i) plot the move-length data as some form of histogram on log-log axes; (ii) draw or fit a straight line across the full range of data or just the tail; (iii) define $\mu$ to be the negative of the slope of the line; (iv) conclude that the data follow a power law of exponent $\mu$ (across the full range or just the tail); (v) then if $1<\mu \leq 3$ conclude that the organism performs a Lévy flight with exponent $\mu$.

It is well known that log-log axes tend to make relationships look straight, and so it is problematic to only plot the data on a log-log plot and then conclude that the data lie on a straight line. One should at least consider an alternative movelength distribution, such as the exponential that corresponds to a simple uncorrelated Poisson random process. Ref. 16 did also test the exponential, though used the unreliable ${ }^{24,25}$ LT method for the power law and compared the distributions 
by comparing coefficients of variation ${ }^{29}\left(R^{2}\right)$, which is not useful for choosing between models ${ }^{19}$.

Here we summarize how to use modern statistical methods of model selection ${ }^{19,20}$ to test whether a given data set $\mathbf{x}=\left\{x_{1}, x_{2}, x_{3}, \ldots, x_{n}\right\}$ provides more evidence for a power-law tail or an exponential tail. Considering the tail to start at $a$, the power-law tail has pdf

$$
f_{1}(x)=C x^{-\mu}, \quad x \geq a,
$$

where the normalization constant $C=(\mu-1) a^{\mu-1}$, and the exponential tail has pdf

$$
f_{2}(x)=\lambda \mathrm{e}^{-\lambda(x-a)}, \quad x \geq a .
$$

The log-likelihood function ${ }^{18,19}$ for the power law is ${ }^{23}$

$$
\log \left[\mathrm{L}_{1}(\mu \mid \text { data } \mathbf{x})\right]=n \log (\mu-1)+n(\mu-1) \log a-\mu \sum_{j=1}^{n} \log x_{j}
$$

where $\mathrm{L}_{1}(\mu \mid$ data $\mathbf{x})$ is the likelihood of a particular value of the unknown parameter $\mu$ given the known data $\mathbf{x}$ (and $\log$ is natural $\log$ ). For the exponential model the unknown parameter is $\lambda$, and

$$
\log \left[\mathrm{L}_{2}(\lambda \mid \text { data } \mathbf{x})\right]=n \log \lambda+n \lambda a-\lambda \sum_{j=1}^{n} x_{j}
$$


Solving for the maximum likelihood estimates (MLEs) analytically ${ }^{23}$ gives $\hat{\mu}=1-n /\left(n \log a-\sum_{j=1}^{n} \log x_{j}\right)$ and $\hat{\lambda}=1 /\left(\sum_{j=1}^{n} x_{j} / n-a\right)$. Akaike's Information Criterion $^{18,19}$ (AIC) for model $i(i=1,2)$ is

$$
\mathrm{AIC}_{i}=-2 \log \left[\mathrm{L}_{i}\left(\hat{\theta}_{i} \mid \text { data } \mathbf{x}\right)\right]+2 K_{i}
$$

where $\hat{\theta_{1}}=\hat{\mu}, \hat{\theta_{2}}=\hat{\lambda}$, and $K_{i}$ is the number of parameters being estimated for model $i\left(K_{1}=K_{2}\right.$ here $)$. The best model is the one with the minimum AIC, $\mathrm{AIC}_{\text {min }}$. Then, AIC differences are given by $\Delta_{i}=\mathrm{AIC}_{i}-\mathrm{AIC}_{m i n}$. The Akaike weights ${ }^{19}$ are relative likelihoods of each model, given by

$$
w_{i}=\frac{\mathrm{e}^{-\Delta_{i} / 2}}{\mathrm{e}^{-\Delta_{1} / 2}+\mathrm{e}^{-\Delta_{2} / 2}},
$$

normalized so the weights sum to 1 . The weight $w_{i}$ is considered as the weight of evidence in favour of model $i$ being the best model for the given data, out of the models considered. Multiple models of varying complexity may also be simultaneously considered ${ }^{19}$. Natural data that follow a power law must be finitely truncated; any approach that neglected this would suffer to some degree (see Supplementary Information). Future work could explore the problem of inference of truncated ${ }^{3}$ Lévy flights.

The likelihood approach clarifies what is meant by the 'tail' of the data (permitting goodness-of-fit tests), eliminates binning problems associated with 
$\log$-log histograms (outlined in ref. 24), and yields 95\% CIs (computed using the profile likelihood-ratio test ${ }^{18}$ ). Furthermore, if the power law is supported by the data, then the MLE for $\mu$ is more accurate (AME, in prep.) than the estimate obtained from the LBN method. 


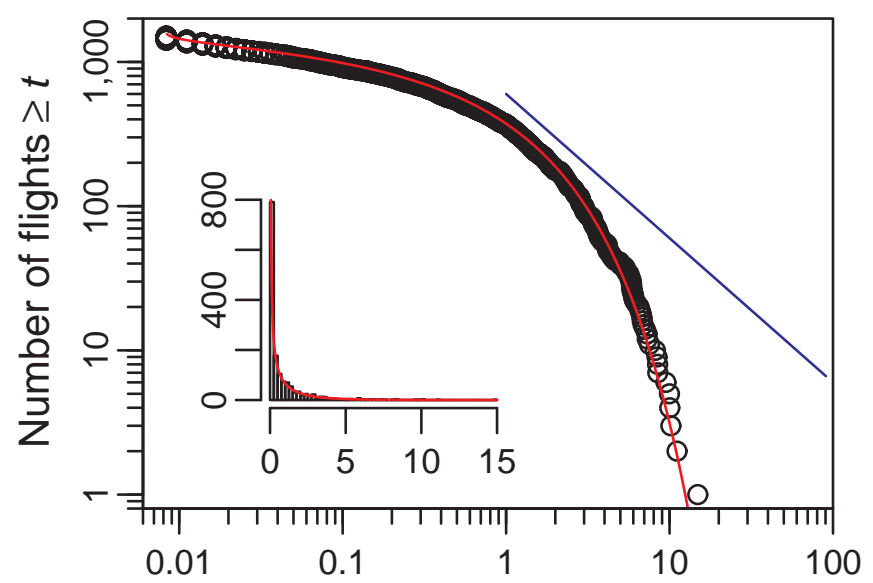

Flight duration, $t, \mathrm{~h}$

Figure 1: 


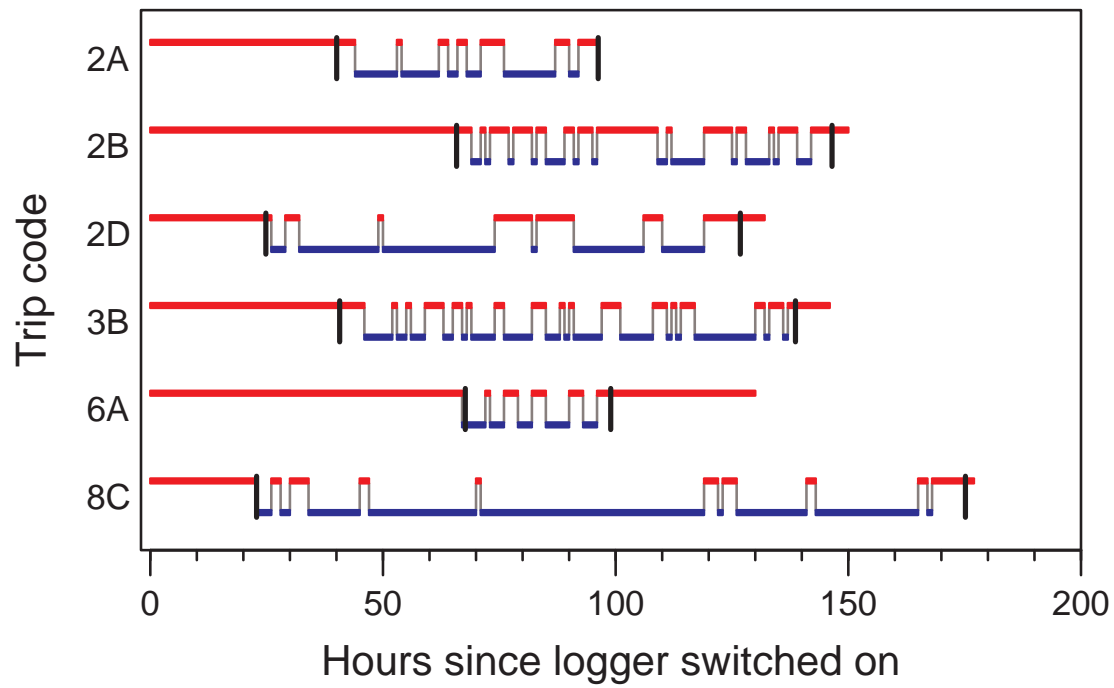

Figure 2: 


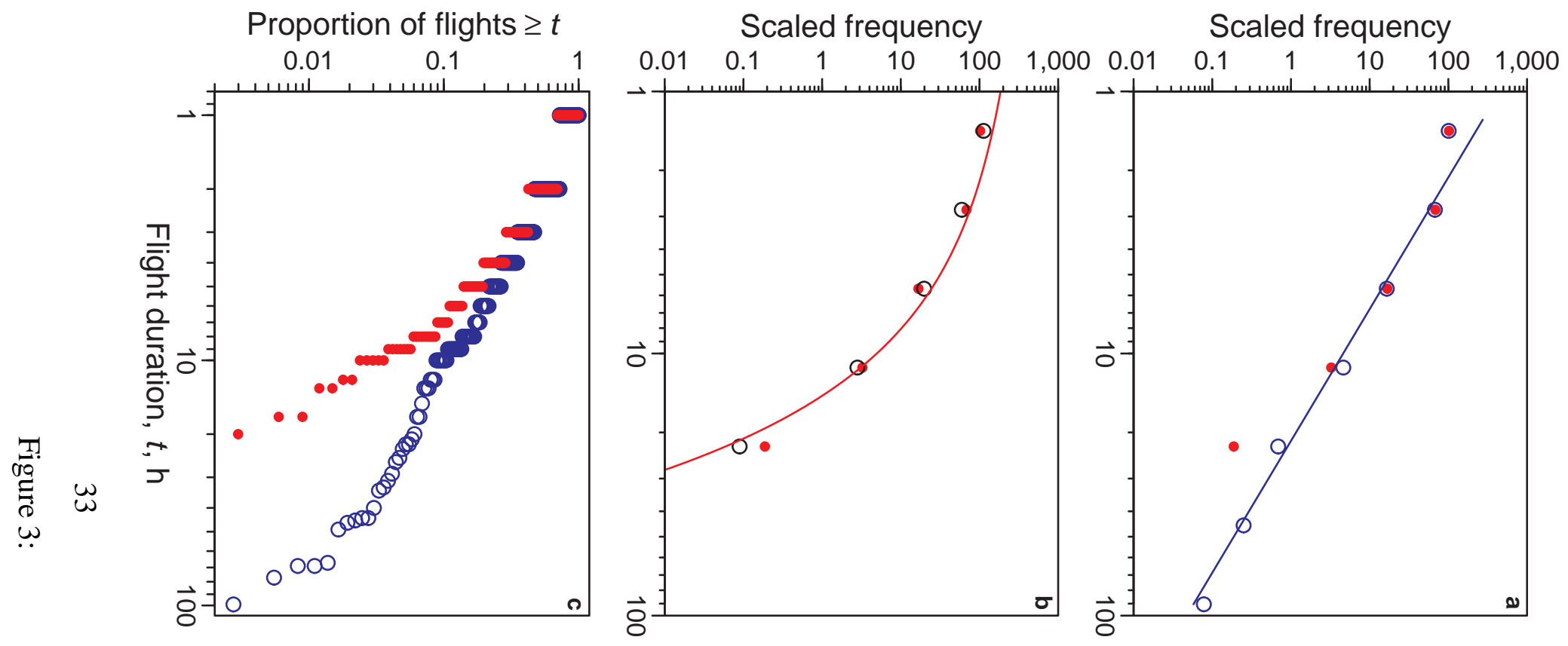



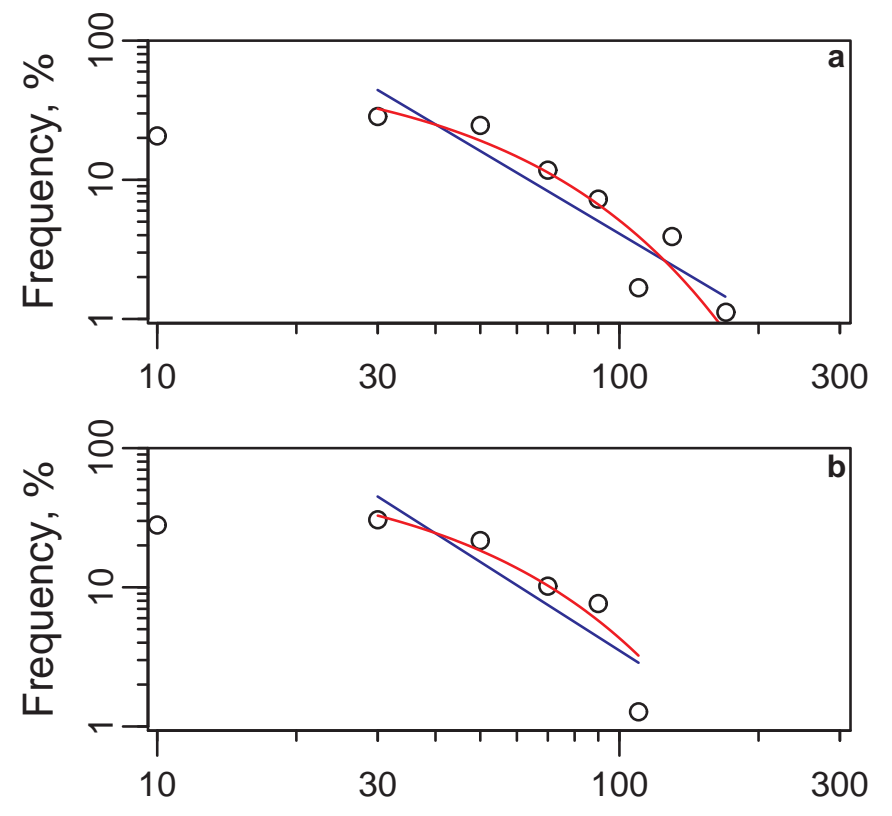

Foraging time, s

Figure 4: 